\title{
Verification and Validation of Knowledge Based Systems
}

\author{
Marc Ayel \\ Universitè de Savoie-Aderias-Lia F-73376 \\ Le Bourget du Lac-France \\ Tel:0033-79758845, Fax:0033-79758785
}

\begin{abstract}
The goal of this talk is to show that the set of available Validation and Verification (V\&V) tools could be larger for Knowledge-Based Systems (KBSs) than for classical software. All the tools which use a black box approach may be applied on KBSs and a great number of glass box tools can be adapted to KBSs. For example the test-data generation is really effective with KBSs.

Moreover, the KBSs have more or less a declarative part and $\mathrm{V} \& \mathrm{~V}$ requirements can be checked on the corresponding Knowledge Bases (KB). The KB consistency can be one of these requirements.

Some of the V\&V requirements are very difficult to check on KBSs. The reason is not in the nature of a KBS or in the nature of the checking. The KBS approach is used to solve complex problems and then the $V \& V$ checkings are obviously also complex problems.
\end{abstract}

